\title{
Enumerating Up-Side Self-Avoiding Walks on Integer Lattices
}

\author{
Lauren K Williams \\ Harvard University \\ Cambridge, MA 02138 \\ lkwill@husc. harvard. edu
}

Submitted: September 4, 1996; Accepted: September 26, 1996.

\begin{abstract}
A self-avoiding walk (saw) is a path on a lattice that does not pass through the same point twice. Though mathematicians have studied saws for over fifty years, the number of $n$-step saws is unknown. This paper examines a special case of this problem, finding the number of $n$ step "up-side" saws (ussaws), saws restricted to moving up and sideways. It presents formulas for the number of $n$-step ussaws on various lattices, found using generating functions with decomposition and recursive methods.
\end{abstract}

\section{Introduction}

A self-avoiding walk (saw) is a path on a lattice that does not cross the same point twice; on the two-dimensional lattice it is a finite sequence of distinct lattice points $\left[\left(x_{0}, y_{0}\right)=(0,0)\right.$, $\left.\left(x_{1}, y_{1}\right), \cdots\left(x_{n}, y_{n}\right)\right]$, such that for all $i,\left(x_{i}, y_{i}\right)$ and $\left(x_{i+1}, y_{i+1}\right)$ are separated by a unit distance. Saws, although easy to describe, pose a number of interesting unsolved problems. In particular, the number of $n$-step saws is unknown.

I began my study of the number of $n$-step saws at the 1994 Research Science Institute by reviewing a paper by Doron Zeilberger, a professor of math at Temple University. He described his approach, studying $n$-step saws on lattices of restricted width [4], as follows: "When a problem seems intractable, it is often a good idea to study 'toy' versions of it in the hope that as the toys become increasingly larger ... they would metamorphose, in the limit, to the real thing."

My analysis also considers a restricted version of the problem of counting saws - counting "up-side" saws (ussaws), saws that

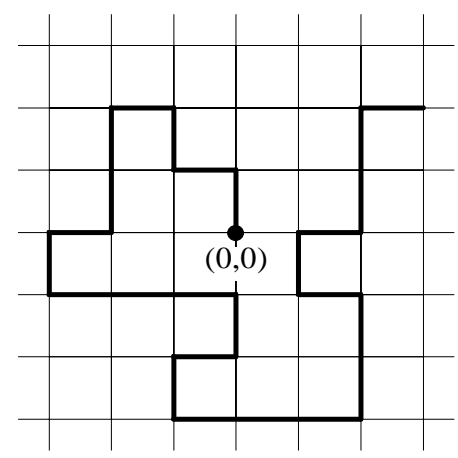

A Self Avoiding Walk move up and sideways but not down, on various lattices. I utilize two methods: an extension of Zeilberger's "decomposition method," and a recursive method.

Generating functions (gf's) are a primary tool of these methods. According to Wilf [3], a generating function is "a clothesline on which we hang up a sequence of numbers for display." It 
is a power series whose coefficients are the successive terms of a sequence, with the coefficient of $t^{i}$ corresponding to the $i$ th element of the sequence. We define $\mathcal{G}_{[m, n]}(t)=\sum_{i=0}^{\infty} a_{i} t^{i}$, where $a_{i}$ is the number of $i$-step ussaws on the $[\mathrm{m}, \mathrm{n}]$ strip, and the $[\mathrm{m}, \mathrm{n}]$ strip is the two-dimensional integer lattice with lateral boundaries $x=m$ and $x=n$.

I determined formulas for the number of $n$-step ussaws on the $[0, \mathrm{~m}]$ strips for $m=1$ to 9 , on the three-dimensional cubic lattice, on the plane, and on the triangular lattice. I also computed the number of ussaws of specified height and length, and found the average height of an $n$-step ussaw.

\section{The Decomposition Method Applied to the [0,1] Strip}

Zeilberger used a decomposition method to find a generating function counting $n$-step saws on the $[0,1]$ strip. We will solve the same problem for ussaws.

Our plan is as follows: first we find a "decomposition," a general sequence of pieces which makes up all ussaws on the given lattice. Then we use gf's to count the number of ways to build each piece. The product of the gf's for each piece is a gf which enumerates $n$-step ussaws on our lattice.

After experimentation, we find that all ussaws that begin at $(0,0)$ on the $[0,1]$ strip can be decomposed into the sequence of pieces $u^{*} L^{*} \tilde{s}$, where the pieces are defined below. Steps up and to the side are denoted by $u$ and $s$ respectively, and the superscripts indicate the number of unit steps taken.

- $u^{*}$ is any number of steps up. (* indicates an indefinite superscript $\geq 0$.)

- $L^{*}$ is any number of $L$ pieces, where $L=s u u^{*}$, a step sideways followed by at least one step up.

- $\tilde{s}$ is an optional step sideways - either a 0 or a 1-step piece. ( $\sim$ indicates that a piece is optional)

The picture illustrates the decomposition of a ussaw into $u^{*} L^{*} \tilde{s}$. This ussaw is coded

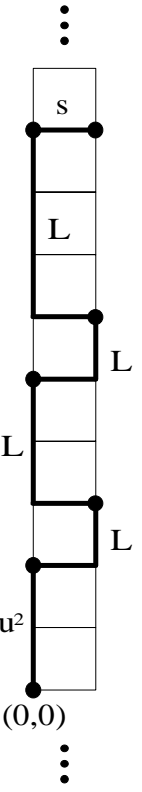
as $u^{2} s u s u^{2} s u s u^{3} s$, equivalent to $\left(u^{2}\right)(s u)\left(s u^{2}\right)(s u)\left(s u^{3}\right)(s)=\left(u^{2}\right)\left(L^{4}\right)(s)=u^{*} L^{*} \tilde{s}$. To see that all ussaws on the $[0,1]$ strip are in the form $u^{*} L^{*} \tilde{s}$, note that an arbitrarily long ussaw contains 0 or more $L$ 's - side-up pieces. Thus any ussaw contains $L^{*}$. A ussaw can begin with an $L$, or, if its first step is not the sideways step of an $L$, it can begin with some steps up. Thus the first piece of a ussaw is $u^{*}$. A ussaw can end with an $L$, or, if its last steps are not the up-steps of an $L$, it can end with a step sideways. Thus the last piece of a ussaw is $\tilde{s}$, an optional step to the side.

Now we find the gf's of each of the pieces.

First, consider $u^{*}$ pieces. There is one 0 -step $u^{*}$ piece (by convention), one 1-step $u^{*}$ piece, and, it is easily seen, one $n$-step $u^{*}$ piece for every $n$. Thus

$$
\mathbf{g f}\left(\mathbf{u}^{*}\right)=\mathbf{1}+\mathbf{t}+\mathbf{t}^{2}+\cdots=\frac{1}{1-\mathbf{t}}
$$

Next, consider $L^{*}$ pieces. Since an $L^{*}$ piece is any number of $L$ 's in a row, $g f\left(L^{*}\right)=g f\left(L^{0}\right)+g f\left(L^{1}\right)+g f\left(L^{2}\right)+\cdots=[g f(L)]^{0}+[g f(L)]^{1}+[g f(L)]^{2}+\cdots=\frac{1}{1-g f(L)} !$

Since every $L$ piece is a step sideways followed by one or more steps up, there is one $n$-step $L$ piece

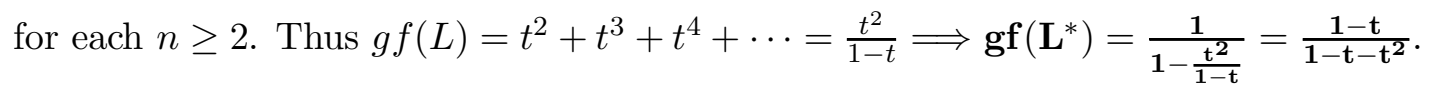




\begin{tabular}{|l|l|l|l|}
\hline Piece & Definition & GF(closed) & GF(expanded) \\
\hline$M_{S}$ & $u^{*} \tilde{s}$ & $\frac{2 t}{1-t}$ & $2 t+2 t^{2}+2 t^{3}+2 t^{4}+\cdots$ \\
\hline$S_{S}$ & $u u^{*} s^{2}$ & $\frac{t^{3}}{1-t}$ & $t^{3}+t^{4}+t^{5}+t^{6}+t^{7}+\cdots$ \\
\hline$S_{S}{ }^{*}$ & & $\frac{1-t}{1-t-t^{3}}$ & $1+t^{3}+t^{4}+t^{5}+2 t^{6}+\cdots$ \\
\hline$\widetilde{S_{M}}$ & $\widehat{u u^{*} s}$ & $\frac{1-t+t^{2}}{1-t}$ & $1+t^{2}+t^{3}+t^{4}+t^{5}+t^{6}+\cdots$ \\
\hline$u$ & $u$ & $t$ & $t$ \\
\hline$u^{*}$ & $u^{*}$ & $\frac{1}{1-t}$ & $1+t+t^{2}+t^{3}+t^{4}+\cdots$ \\
\hline$\left[S_{S}{ }^{*} S_{M} u M_{S}\right]^{*}$ & & $\frac{(1-t)\left(1-t-t^{3}\right)}{1-2 t+t^{2}-t^{3}-t^{4}}$ & $1+2 t^{4}+4 t^{5}+6 t^{6}+10 t^{7}+\cdots$ \\
\hline
\end{tabular}

Table 1: Pieces on the $[-1,1]$ Strip

Finally, consider $\tilde{s}$ pieces. There is one 0 -step $\tilde{s}$ piece and one 1 -step $\tilde{s}$ piece. Thus $\mathbf{g f}(\tilde{\mathbf{s}})=\mathbf{1}+\mathbf{t}$. The gf of ussaws in the form $u^{*} L^{*} \tilde{s}$ is equal to $\left(g f\left(u^{*}\right)\right)\left(g f\left(L^{*}\right)\right)(g f(\tilde{s}))=\left(\frac{1}{1-t}\right)\left(\frac{1-t}{1-t-t^{2}}\right)(1+t)$, so

$$
\mathcal{G}_{[0,1]}(t)=\frac{1+t}{1-t-t^{2}}=1+2 t+3 t^{2}+5 t^{3}+8 t^{4}+13 t^{5} \cdots
$$

This is actually the generating function for the Fibonacci numbers!

\section{The Decomposition Method Applied to Other Lattice Strips}

The method shown in $\S 2$ can be extended to the [-1,1], [0,2], and $[0,3]$ strips.

Ussaws on the [-1,1] strip are in one of the forms: $u^{*}$ and $M_{S}\left[S_{S}{ }^{*} S_{M} u M_{S}\right]^{*} S_{S}{ }^{*} \widetilde{S_{M}} u^{*}$. The pieces are defined as follows: $M_{S}$ takes 0 or more steps up followed by a step sideways; $S_{S}$ takes at least one step up followed by two steps sideways; and $S_{M}$ takes at least one step up followed by a step sideways. All three pieces start and end at the lines indicated by the two letters. $M$ refers to "middle," the line $x=0$, and $S$ refers to "side," the lines $x=-1$ and $x=1$. As before, $u$ and $s$ indicate unit steps up and sideways.

The picture illustrates a ussaw which is coded as $M_{S}\left[S_{M} u M_{S}\right]\left[S_{S}{ }^{2} S_{M} u M_{S}\right] S_{S} S_{M} u^{2}=$ $M_{S}\left[S_{S}{ }^{*} S_{M} u M_{S}\right]^{2} S_{S} S_{M} u^{2}=M_{S}\left[S_{S}{ }^{*} S_{M} u M_{S}\right]^{*} S_{S}{ }^{*} \widetilde{S_{M}} u^{*}$.

Substituting our gf's into $u^{*}$ and $M_{S}\left[S_{S}{ }^{*} S_{M} u M_{S}\right]^{*} S_{S}{ }^{*} \widetilde{S_{M}} u^{*}$ yields:

$$
\begin{gathered}
\frac{1}{1-t}+\frac{2 t}{1-t} \cdot \frac{(1-t)\left(1-t-t^{3}\right)}{1-2 t+t^{2}-t^{3}-t^{4}} \cdot \frac{1-t}{1-t-t^{3}} \cdot \frac{1-t+t^{2}}{1-t} \cdot \frac{1}{1-t}=\frac{1+t+t^{3}}{1-2 t+t^{2}-t^{3}-t^{4}} . \\
\therefore \mathcal{G}_{[-1,1]}(t)=\frac{1+t+t^{3}}{1-2 t+t^{2}-t^{3}-t^{4}}=1+3 t+5 t^{2}+9 t^{3}+17 t^{4}+\cdots
\end{gathered}
$$

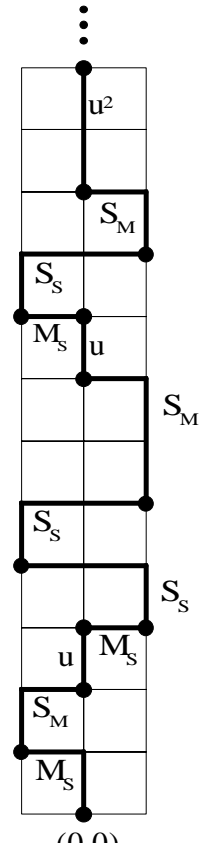

$(0,0)$ 


\begin{tabular}{|l|l|l|l|}
\hline Piece & Definition & GF(closed) & GF(expanded) \\
\hline$u^{*}$ & $u^{*}$ & $\frac{1}{1-t}$ & $1+t+t^{2}+t^{3}+t^{4} \cdots$ \\
\hline$\widetilde{O_{O}}$ & $\widetilde{s}$ & $1+t^{3}$ & $1+t^{3}$ \\
\hline$O_{O}{ }^{*}$ & $\left(u u^{*} s^{3}\right)^{*}$ & $\frac{1-t}{1-t-t^{4}}$ & $1+t^{4}+t^{5}+t^{6}+\cdots$ \\
\hline$O_{I}$ & $s$ or $s^{2}$ & $t+t^{2}$ & $t+t^{2}$ \\
\hline$I_{I}{ }^{*}$ & $\left(u u^{*} s\right)^{*}$ & $\frac{1-t}{1-t-t^{2}}$ & $1+t^{2}+t^{3}+2 t^{4} \cdots$ \\
\hline$I_{O}$ & $u u^{*} s$ or $u u^{*} s^{2}$ & $\frac{t^{2}+t^{3}}{1-t}$ & $t^{2}+2 t^{3}+2 t^{4}+2 t^{5} \cdots$ \\
\hline$A$ & {$\left[O_{O}{ }^{*} O_{I} I_{I} I_{O}\right]^{*} O_{O}{ }^{*} \widetilde{O}_{I} I_{I}{ }^{*} u^{*}$} & $\frac{\left(1-t-t^{2}\right)\left(1-t-t^{4}\right)}{1-2 t+t^{3}-2 t^{4}-t^{5}}$ & $1+t^{4}+4 t^{5}+9 t^{6} \cdots$ \\
\hline
\end{tabular}

Table 2: Pieces on the [0,3] Strip

$\mathcal{G}_{[0,2]}(t)$ is similar to $\mathcal{G}_{[-1,1]}(t)$ since both strips have the same width. The difference arises because $(0,0)$ is at the side of the $[0,2]$ strip but at the middle of the $[-1,1]$ strip.

$$
\mathcal{G}_{[0,2]}(t)=\frac{1+t^{2}+t^{3}}{1-2 t+t^{2}-t^{3}-t^{4}}=1+2 t+4 t^{2}+8 t^{3}+15 t^{4}+\cdots
$$

On the $[0,3]$ strip all ussaws are in one of the forms: $\widetilde{O_{O}} A, O_{I} I_{I}{ }^{*} I_{O} A$, and $O_{I} I_{I}{ }^{*} u^{*}$.

Besides $A$, the pieces start and end at the lines indicated by the two letters, where $O$ refers to the outer lines of the lattice strip (the lines $x=0$ and $x=3$ ) and $I$ refers to the inner lines. See Table 2 for the definitions and gf's. Adding the products of the gf's corresponding to the three sequences of pieces yields:

$$
\mathcal{G}_{[0,3]}(t)=\frac{(1+t)\left(1-t+t^{2}+t^{3}\right)}{1-2 t+t^{3}-2 t^{4}-t^{5}}=1+2 t+4 t^{2}+9 t^{3}+19^{4}+\cdots
$$

\section{A Generalization of the Decomposition Method}

We would like to find a more general technique for finding $\mathcal{G}_{[0, n]}(t)$; this section describes a method for finding $\mathcal{G}_{[0, n+1]}(t)$, given $\mathcal{G}_{[0, n]}(t)$. 


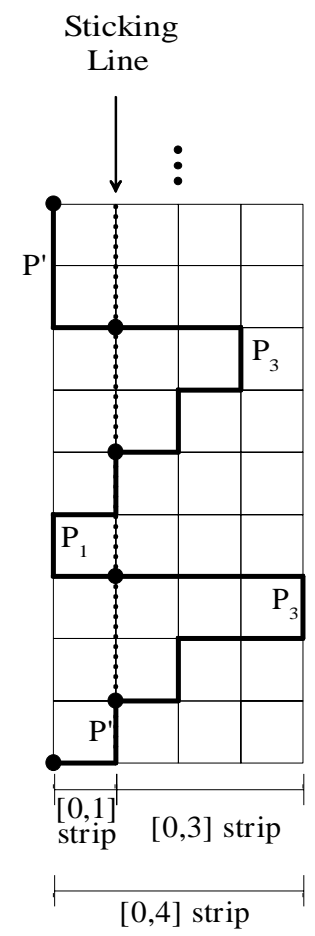

Consider a ussaw on the $[0, \mathrm{n}+1]$ strip and think of the $[0, \mathrm{n}+1]$ strip as a $[0,1]$ strip stuck to a $[0, \mathrm{n}]$ strip, so that the line $x=1$ on the $[0,1]$ strip coincides with the line $x=0$ on the $[0, \mathrm{n}]$ strip. Call this line the "sticking line." Call all lines parallel to this line (including the sticking line itself) verticals. As shown in the diagram, any ussaw on the $[0, n+1]$ strip can be decomposed into a sequence of pieces: a piece on the $[0,1]$ strip, followed by a piece on the $[0, n]$ strip, followed by a piece on the $[0,1]$ strip ...

In order to determine where a piece begins and ends, we specify that: a piece on the $[0,1]$ or $[0, n]$ strip is "ended" only if the following step goes to a point on the other strip not on the sticking line; every piece in the sequence, besides the first and last, begins and ends on the "sticking line"; a new piece begins at the endpoint of the previous piece.

These conditions uniquely determine the endpoints of each piece. They also determine that successive pieces, other than the first and last, must begin with a step sideways. We now define: - $P_{n}$ is a piece on the $[0, \mathrm{n}]$ strip which begins and ends at the same vertical, and begins with a step sideways.

- $P^{\prime}$ is a piece on the $[0,1]$ strip which begins and ends at opposite verticals, and begins with a step sideways.

By experimentation we find that $P_{n+1}$ is in one of the forms: $P_{1}$ and $P^{\prime}\left(P_{n} P_{1}\right)^{*} P_{n} P^{\prime}$. In the picture, a $P_{n+1}$ piece is decomposed as $P^{\prime} P_{3} P_{1} P_{3} P^{\prime}$, equivalent to $P^{\prime} P_{n} P_{1} P_{n} P^{\prime}=P^{\prime}\left(P_{n} P_{1}\right)^{1} P_{n} P^{\prime}=$ $P^{\prime}\left(P_{n} P_{1}\right) * P_{n} P^{\prime}$.

Thus $g f\left(P_{n+1}\right)=g f\left(P_{1}\right)+g f\left[P^{\prime}\left(P_{n} P_{1}\right)^{*} P_{n} P^{\prime}\right]$. It is fairly simple to compute that $g f\left(P_{1}\right)=$ $\frac{t^{3}}{1-2 t+t^{2}-t^{4}}$ and $g f\left(P^{\prime}\right)=\frac{t(1-t)}{1-2 t+t^{2}-t^{4}}$. We can find $g f\left(P_{2}\right)$ by letting $n=1$ in the above formula. Once we have $g f\left(P_{2}\right)$ we can find $g f\left(P_{3}\right)$, and then $g f\left(P_{4}\right) \ldots$ Theoretically, we can find $g f\left(P_{n}\right)$ 
for any $n$. And given $g f\left(P_{n}\right)$, we can find $\mathcal{G}_{[0, n]}(t)$ : if $S_{n}$ is the sequence of pieces that describes ussaws on the $[0, \mathrm{n}]$ strip, $S_{n}$ is either in the form $U^{*} P_{n}\left(\widetilde{u P^{\prime} S_{n-1}}\right)$ or the form $u^{*}\left(\widetilde{P^{\prime} S_{n-1}}\right)$.

\section{The Decomposition Method Applied to the Cubic Lattice}

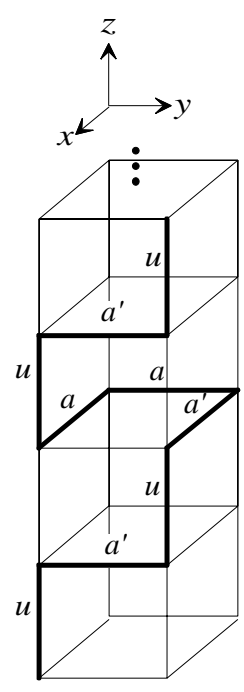

$(0,0,0)$

The decomposition method can be applied to a special case of the three-dimensional cubic lattice, the lattice restricted in both the $x$ and $y$-dimensions to $[0,1]$, but infinite in the $z$-dimension. Saws on this lattice are in the form: $u^{*}\left[a^{\prime} a^{m} u u^{*}\right]^{*} \widehat{a^{\prime} a^{m}}$ where $m=0,1$, or 2 .

The gf's for $u, u^{*}, a^{\prime}, a$, and $a^{m}$ are: $t, \frac{1}{1-t}, 2 t, t$, and $1+t+t^{2}$.

Here, $u$ is a step "up," parallel to the $z$-axis, while $a^{\prime}$ and $a$ are steps "across," parallel to either of the other two axes. An $a^{\prime}$ must be the first step taken or must follow a step up, so it always has two choices of direction. However, an $a$ must follow another step across and so its direction is determined by the previous step.

The picture illustrates a cubic lattice with a ussaw coded as $u a^{\prime} a^{2} u a^{\prime} u a^{\prime} u a^{\prime} a^{2} u a^{\prime} u$, equivalent to $u\left(a^{\prime} a^{2} u\right)\left(a^{\prime} u\right)\left(a^{\prime} u\right)\left(a^{\prime} a^{2} u\right)\left(a^{\prime} u\right)=u\left(a^{\prime} a^{m} u u^{*}\right)^{5}=u^{*}\left(a^{\prime} a^{m} u u^{*}\right)^{*} \widehat{a^{\prime} a^{m}}$.

Substituting the gf's into $u^{*}\left[a^{\prime} a^{m} u u^{*}\right]^{*} \widehat{a^{\prime} a^{m}}$ yields:

$$
\mathcal{G}(t)=\frac{1+2 t+2 t^{2}+2 t^{3}}{1-t-2 t^{2}-2 t^{3}-2 t^{4}}=1+3 t+7 t^{2}+17 t^{3}+39 t^{4}+\cdots
$$

\section{The Recursive Method for Enumerating Saws on the [0,1] Strip}
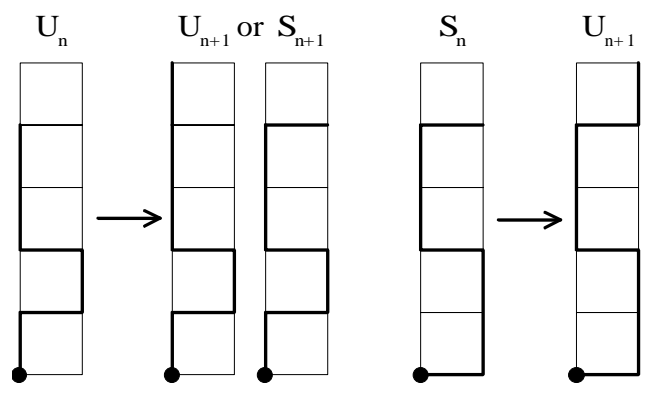

This method utilizes the fact that an $(n+1)$-step ussaw is formed by adding one step to an $n$-step ussaw.

Let $U_{n}$ and $S_{n}$ be $n$-step ussaws ending with a step up and sideways, respectively, and let $u_{n}$ and $s_{n}$ be the numbers of $U_{n}$ 's and $S_{n}$ 's. Let $\mathcal{U}_{[0,1]}(t)=\sum_{n=0}^{\infty} u_{n} t^{n}$, $\mathcal{S}_{[0,1]}(t)=\sum_{n=0}^{\infty} s_{n} t^{n}$, and $\mathcal{G}_{[0,1]}(t)=\sum_{n=0}^{\infty} g_{n} t^{n}$, where $g_{n}$ is the total number of $n$-step ussaws.

Note that by adding a step to a $U_{n}$ we can form either a $U_{n+1}$ or an $S_{n+1}$, and by adding a step to an $S_{n}$ we can form a $U_{n+1}$. Moreover, every $U_{n+1}$ is a $U_{n}$ or an $S_{n}$ with an extra up-step, and an $S_{n+1}$ is a $U_{n}$ with an extra side-step. Thus, $u_{n+1}=u_{n}+s_{n}$ and $s_{n+1}=u_{n}$, which lead to $u_{n+2}=u_{n+1}+u_{n}$. Multiplying by $t^{n}$, we get $u_{n+2} t^{n}=u_{n+1} t^{n}+u_{n} t^{n}$. We now use a summation technique to solve for $\mathcal{U}_{[0,1]}(t)$ : 


$$
\begin{aligned}
\sum_{n=0}^{\infty} u_{n+2} t^{n} & =\sum_{n=0}^{\infty} u_{n+1} t^{n}+\sum_{n=0}^{\infty} u_{n} t^{n} \\
\frac{u_{2} t^{2}+u_{3} t^{3}+u_{4} t^{4}+\cdots}{t^{2}} & =\frac{u_{1} t+u_{2} t^{2}+u_{3} t^{3}+\cdots}{t}+\mathcal{U}_{[0,1]}(t) \\
\frac{\mathcal{U}_{[0,1]}(t)-u_{1} t-u_{0}}{t^{2}} & =\frac{\mathcal{U}_{[0,1]}(t)-u_{0}}{t}+\mathcal{U}_{[0,1]}(t) .
\end{aligned}
$$

By substituting $u_{0}=1$ and $u_{1}=1$ into the above, we find that $\mathcal{U}_{[0,1]}(t)=\frac{1}{1-t-t^{2}}$. Similarly we get $\mathcal{S}_{[0,1]}(t)=\frac{t}{1-t-t^{2}}$. Confirming our results in $\S 2$, we find that $\mathcal{G}_{[0,1]}(t)=\mathcal{U}_{[0,1]}(t)+\mathcal{S}_{[0,1]}(t)=$ $\frac{1+t}{1-t-t^{2}}$.

To obtain the $n$th term formula, we use the method of characteristic equations. Recall that $u_{n+2}=u_{n+1}+u_{n}$. The corresponding characteristic equation is $x^{2}=x+1$, with roots $r_{1}, r_{2}=\frac{1 \pm \sqrt{5}}{2}$. Thus $u_{n}=K_{1} r_{1}^{n}+K_{2} r_{2}^{n}$, where $K_{1}$ and $K_{2}$ are real. Since $s_{n+1}=u_{n}, s_{n}=K_{1} r_{1}^{n-1}+K_{2} r_{2}^{n-1}$. Substituting small values of $u_{n}$ and $s_{n}$ into the above expressions, we get $\mathcal{F}_{[0,1]}(n)=u_{n}+s_{n}=$ $\left(\frac{1}{\sqrt{5}}\right)\left[\left(\frac{1+\sqrt{5}}{2}\right)^{n+2}-\left(\frac{1-\sqrt{5}}{2}\right)^{n+2}\right]$.

\section{A Generalization of the Recursive Method}

We introduce some new notation in order to generalize the method. On the $[0, \max ]$ strip, we refer to the lines parallel to the $y$-axis as verticals, and number them from left to right, from 0 to $\max$.

Let $\left(U_{i}\right)_{n},\left(L_{i}\right)_{n}$, and $\left(R_{i}\right)_{n}$ be $n$-step ussaws that end with a step up along the $i$ th vertical, a step left to the $i$ th vertical, and a step right to the $i$ th vertical. Let $\left(u_{i}\right)_{n},\left(l_{i}\right)_{n}$, and $\left(r_{i}\right)_{n}$ be the numbers of $\left(U_{i}\right)_{n}$ 's, $\left(L_{i}\right)_{n}$ 's, and $\left(R_{i}\right)_{n}$ 's, respectively. The following recurrences hold:

- $\left(u_{i}\right)_{n+1}=\left(u_{i}\right)_{n}+\left(r_{i}\right)_{n}+\left(l_{i}\right)_{n}$ for every $i$ from 0 to $\max$.

- $\left(l_{i}\right)_{n+1}=\left(u_{i+1}\right)_{n}+\left(l_{i+1}\right)_{n}$ for every $i$ from 0 to max.

- $\left(r_{i}\right)_{n+1}=\left(u_{i-1}\right)_{n}+\left(r_{i-1}\right)_{n}$ for every $i$ from 0 to $\max$.

We can solve the recurrences for any value of $\max$. Note that there are no ussaws that end with a step right to the 0th vertical or left to

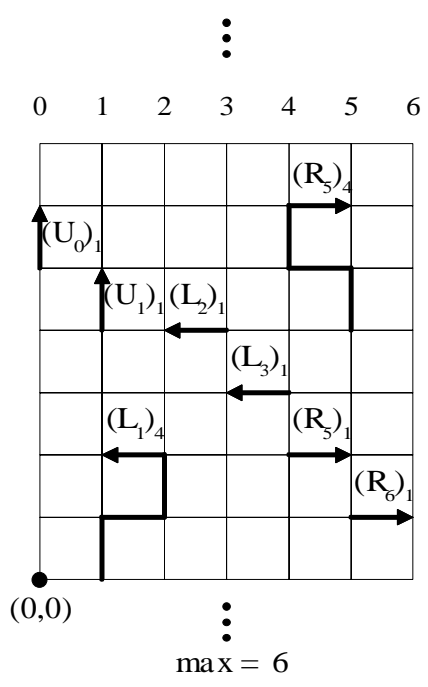
the maxth vertical, so $\left(r_{0}\right)_{n}=\left(l_{\max }\right)_{n}=0$ for all $n$.

\section{The Recursive Method Applied to the Plane}

The recursive method can be used to find a gf $\mathcal{G}_{\square}(t)$ and an $n$th term formula $\mathcal{F}_{\square}(n)$ for the number of $n$-step ussaws on the plane. Let $U_{n}$ and $S_{n}$ be $n$-step ussaws ending with steps up and sideways, respectively, and let $u_{n}$ and $s_{n}$ be the numbers of $U_{n}$ 's and $S_{n}$ 's. Then $u_{n+1}=u_{n}+s_{n}$ and $s_{n+1}=2 u_{n}+s_{n}$, which lead to: 


$$
\begin{aligned}
\mathcal{G}_{\square}(t) & =\frac{1+t}{1-2 t-t^{2}}=1+3 t+7 t^{2}+17 t^{3}+41 t^{4}+\cdots . \\
\mathcal{F}_{\square}(n) & =\frac{1}{2}\left[(1+\sqrt{2})^{n+1}+(1-\sqrt{2})^{n+1}\right] . \\
\mathcal{F}_{\square}(n) & \approx \frac{1}{2}(1+\sqrt{2})^{n+1}
\end{aligned}
$$

\section{The Recursive Method Applied to the Triangular Lattice}

The recursive method can be used to find both a gf $\mathcal{G}_{\triangle}(t)$ and an $n$th term formula $\mathcal{F}_{\triangle}(n)$ for the number of $n$-step ussaws on the triangular lattice. The diagram illustrates two ussaws on this lattice. Let $U_{n}$ be an $n$-step ussaw ending with a step diagonally upwards and let $S_{n}$ be an $n$ step ussaw ending with a step sideways. Let $u_{n}$ and $s_{n}$ be the numbers of $U_{n}$ 's and $S_{n}$ 's. Then $u_{n+1}=2 u_{n}+2 s_{n}$ and $s_{n+1}=2 u_{n}+s_{n}$. Solving these recurrences, we get:

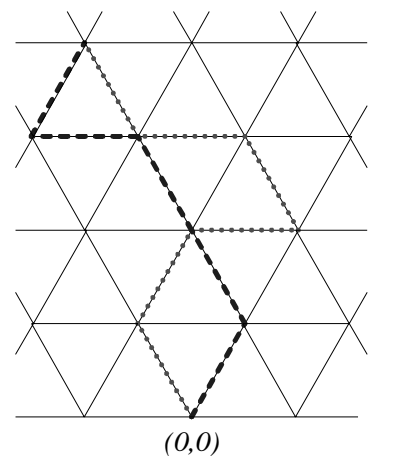

$$
\begin{aligned}
\mathcal{G}_{\triangle}(t) & =\frac{1+t}{1-3 t-2 t^{2}}=1+4 t+14 t^{2}+50 t^{3}+\cdots \\
\mathcal{F}_{\triangle}(n) & =\left(\frac{5 \sqrt{17}+17}{34}\right)\left(\frac{3+\sqrt{17}}{2}\right)^{n}-\left(\frac{5 \sqrt{17}-17}{34}\right)\left(\frac{3-\sqrt{17}}{2}\right)^{n} . \\
\mathcal{F}_{\triangle}(n) & \approx\left(\frac{5 \sqrt{17}+17}{34}\right)\left(\frac{3+\sqrt{17}}{2}\right)^{n} .
\end{aligned}
$$

\section{The Number of $N$-Step Saws of Fixed Height}

Using gf's in two variables, we will find the number of ussaws of height $m$ and length $n$ on the plane, where the height of a ussaw is defined to be the $y$-coordinate of its endpoint. Defining $g(m, n)$ to be the number of ussaws of height $m$ and length $n$, we will solve for:

$$
\mathcal{G}(t, v)=\sum_{m, n=0}^{\infty} g(m, n) t^{m} v^{n}
$$

From now on we will leave out the limits of summation when we are summing over $m$ and $n$, but it is to be understood that the limits on both variables are 0 and $\infty$.

Define $\mathcal{U}(t, v)=\sum u(m, n) t^{m} v^{n}$ and $\mathcal{S}(t, v)=\sum s(m, n) t^{m} v^{n}$, where $u(m, n)$ and $s(m, n)$ are the numbers of ussaws of height $m$ and length $n$ that end with steps up, and sideways. Then

$u(m, n)=u(m-1, n-1)+s(m-1, n-1)$ and $s(m, n)=2 u(m, n-1)+s(m, n-1)$. From the first equation we find that $s(m, n)=u(m+1, n+1)-u(m, n)$. Substituting into the second and 
simplifying, we get $u(m+1, n+2)-u(m+1, n+1)-u(m, n+1)-u(m, n)=0$. Multiplying by $t^{m} v^{n}$ and summing yields:

$\frac{\sum u(m+1, n+2) t^{m+1} v^{n+2}}{t v^{2}}-\frac{\sum u(m+1, n+1) t^{m+1} v^{n+1}}{t v}-\frac{\sum u(m, n+1) t^{m} v^{n+1}}{v}-\mathcal{U}(t, v)=0$,

$\Longleftrightarrow[\mathcal{U}(t, v)-$ terms where the exponent of $\mathrm{t}$ is 0 or exp. of $\mathrm{v}$ is 0 or 1$]-v[\mathcal{U}(t, v)-$ terms where exp. of $\mathrm{t}$ or $\mathrm{v}$ is 0$]$ $-t v[\mathcal{U}(t, v)-$ terms where exp. of $\mathrm{v}$ is 0$]-t v^{2} \mathcal{U}(t, v)=0$.

Only two ussaws end with a step up and have length or height equal to 0 or 1: the 0 -step ussaw and the ussaw composed of one step up. So the only terms where the exponent of $t$ or $v$ is 0 or 1 , are 1 and $t v$. Now:

$$
\begin{aligned}
{[\mathcal{U}(t, v)} & -t v-1]-v[\mathcal{U}(t, v)-1]-t v[\mathcal{U}(t, v)-1]-t v^{2} \mathcal{U}(t, v)=0 \\
& \Longrightarrow \mathcal{U}(t, v)\left[1-v-t v-t v^{2}\right]-t v-1+v+t v=0 \\
& \Longrightarrow \mathcal{U}(t, v)=\frac{1-v}{1-v-t v-t v^{2}}
\end{aligned}
$$

Similarly we find that

$$
\begin{aligned}
\mathcal{S}(t, v) & =\frac{2 v}{1-v-t v-t v^{2}} . \\
\therefore \mathcal{G}(t, v) & =\mathcal{U}(t, v)+\mathcal{S}(t, v)=\frac{1+v}{1-v-t v-t v^{2}} .
\end{aligned}
$$

We now find an explicit formula $\mathcal{F}(a, b)$ for the coefficient of the $t^{a} v^{b}$ term, the number of ussaws of height $a$ and length $b$. Note that:

$$
\begin{aligned}
\frac{1+v}{1-v-t v-t v^{2}} & =(1+v)\left[\frac{1}{1-\left(v+t v+t v^{2}\right)}\right] \\
& =(1+v) \sum_{i=0}^{\infty}\left(v+t v+t v^{2}\right)^{i} \\
& =(1+v) \sum_{i=0}^{\infty}\left(v^{i}\right)[1+t(1+v)]^{i} \\
& =(1+v) \sum_{i=0}^{\infty}\left(v^{i}\right) \sum_{j=0}^{i}\left(\begin{array}{l}
i \\
j
\end{array}\right) t^{j}(1+v)^{j} .
\end{aligned}
$$

The portion of the gf in which the exponent of $t$ is fixed at $a$ is:

$$
(1+v) \sum_{i=0}^{\infty}\left(v^{i}\right)\left(\begin{array}{c}
i \\
a
\end{array}\right) t^{a}(1+v)^{a}=t^{a}(1+v)^{a+1} \sum_{i=0}^{\infty}\left(\begin{array}{c}
i \\
a
\end{array}\right)\left(v^{i}\right)=t^{a}(1+v)^{a+1} \cdot \frac{v^{a}}{(1-v)^{a+1}}
$$


Thus the gf for ussaws of fixed height and variable length is:

$$
\mathcal{G}_{a}(v)=\frac{v^{a}(1+v)^{a+1}}{(1-v)^{a+1}}
$$

When expanded, the coefficient of $v^{i}$ gives the number of ussaws of height $a$ and length $i$.

Now we will solve for the coefficient of $t^{a} v^{b}$ in $t^{a}(1+v)^{a+1} \sum_{i=0}^{\infty}\left(\begin{array}{l}i \\ a\end{array}\right)\left(v^{i}\right)$, the number of ussaws of height $a$ and length $b$. This expression is equivalent to $t^{a} \sum_{j=0}^{a+1}\left(\begin{array}{c}a+1 \\ j\end{array}\right) v^{j} \sum_{i=0}^{\infty}\left(\begin{array}{c}i \\ a\end{array}\right)\left(v^{i}\right)$, in which the exponent of $v$ is $j+i$. Let $j+i=b$ and our expression becomes: $t^{a} \sum_{j=0}^{a+1}\left(\begin{array}{c}a+1 \\ j\end{array}\right) v^{j}\left(\begin{array}{c}b-j \\ a\end{array}\right)\left(v^{b-j}\right)$ $=t^{a} v^{b} \sum_{j=0}^{a+1}\left(\begin{array}{c}a+1 \\ j\end{array}\right)\left(\begin{array}{c}b-j \\ a\end{array}\right)$.

$$
\therefore \mathcal{F}(a, b)=\sum_{j=0}^{a+1}\left(\begin{array}{c}
a+1 \\
j
\end{array}\right)\left(\begin{array}{c}
b-j \\
a
\end{array}\right) \text {. }
$$

Similarly to the way we found $\mathcal{G}(t, v)$, we can find a gf $\hat{\mathcal{G}}(t, v)=\sum \hat{g}(m, n) t^{m} v^{n}$, where $\hat{g}(m, n)$ is the number of ussaws of width $m$ and length $n$ on the plane, and the width of a ussaw is defined to be the $x$-coordinate of its endpoint. It turns out that:

$$
\hat{\mathcal{G}}(t, v)=\frac{t\left(1-v^{2}\right)}{t\left(1+v^{2}+v^{3}\right)-v\left(1+t+t^{2}\right)} .
$$

\section{The Average Height of an $N$-Step Saw on the [0,1] Strip}

Using gf's in two variables we can compute the average height of an $n$-step ussaw on the [0,1] strip.

Let $u(m, n)$ and $s(m, n)$ be the numbers of ussaws of height $m$ and length $n$ that end with steps up, and sideways, respectively, and let $g(m, n)$ be the total number of ussaws of height $m$ and length $n$. Define $\mathcal{U}_{n}(t)=\sum_{m=0}^{\infty} u(m, n) t^{m}, \mathcal{S}_{n}(t)=\sum_{m=0}^{\infty} s(m, n) t^{m}$, and $\mathcal{G}_{n}(t)=\sum_{m=0}^{\infty} g(m, n) t^{m}$.

If we have $\mathcal{G}_{n}(t)=\sum_{m=0}^{\infty} g(m, n) t^{m}$, a gf for ussaws of fixed length $n$ and varying height $m$, then the average height of an $n$-step ussaw is:

$$
\frac{\sum m \cdot g(m, n)}{\text { total number of } n \text {-step ussaws }}=\frac{\sum m \cdot g(m, n)}{\sum g(m, n)}=\frac{\mathcal{G}_{n}{ }^{\prime}(1)}{\mathcal{G}_{n}(1)} .
$$

We now solve for $\mathcal{G}_{n}(t)$. Clearly $u(m, n)=u(m-1, n-1)+s(m-1, n-1)$ and $s(m, n)=$ $u(m, n-1)$, from which we get $u(m+1, n+2)-u(m, n+1)-u(m, n)=0$. It follows that:

$$
\begin{gathered}
\frac{\sum_{m=0}^{\infty} u(m+1, n+2) t^{m+1}}{t}-\sum_{m=0}^{\infty} u(m, n+1) t^{m}-\sum_{m=0}^{\infty} u(m, n) t^{m}=0 \\
\frac{\mathcal{U}_{n+2}(t)-\text { terms where exp. of t is } 0}{t}-\mathcal{U}_{n+1}(t)-\mathcal{U}_{n}(t)=0 .
\end{gathered}
$$


But there are no terms where the exponent of $t$ is 0 because $n \geq 0 \Rightarrow n+2 \geq 0 \Rightarrow$ length of ussaw $\geq 2 \Rightarrow$ height $>0$. So $\mathcal{U}_{n+2}(t)=t\left[\mathcal{U}_{n+1}(t)+\mathcal{U}_{n}(t)\right]$. After experimentation we find that $\mathcal{U}_{0}(t)=1, \mathcal{U}_{1}(t)=t, \mathcal{U}_{2}(t)=t^{2}+2, \mathcal{U}_{3}(t)=t^{3}+2 t^{2}$, and by induction we can prove that $\mathcal{U}_{n}(t)=\sum_{i=0}^{n}\left(\begin{array}{c}n-i \\ i\end{array}\right) t^{n-i}$.

To solve for $\mathcal{G}_{n}(t)$ we note that $u(m+1, n+1)=u(m, n)+s(m, n) \Rightarrow \frac{\sum_{m=0}^{n} u(m+1, n+1) t^{m+1}}{t}=$ $\mathcal{U}_{n}(t)+\mathcal{S}_{n}(t) \Rightarrow \frac{\mathcal{U}_{n+1}(t)}{t}=\mathcal{U}_{n}(t)+\mathcal{S}_{n}(t)$. But $\mathcal{U}_{n}(t)+\mathcal{S}_{n}(t)=\mathcal{G}_{n}(t)$ ! Thus $\mathcal{G}_{n}(t)=\frac{\mathcal{U}_{n+1}(t)}{t}$.

$$
\begin{gathered}
\mathcal{G}_{n}(t)=\sum_{i=0}^{n}\left(\begin{array}{c}
n+1-i \\
i
\end{array}\right) t^{n-i}=\sum_{m=0}^{n}\left(\begin{array}{c}
m+1 \\
n-m
\end{array}\right) t^{m} . \\
\mathcal{F}(m, n)=\left(\begin{array}{c}
m+1 \\
n-m
\end{array}\right) .
\end{gathered}
$$

Thus there are $\left(\begin{array}{c}m+1 \\ n-m\end{array}\right)$ ussaws of height $m$ and length $n$ on the $[0,1]$ strip.

Now $\mathcal{G}_{n}(1)=\sum_{i=0}^{n}\left(\begin{array}{c}n+1-i \\ i\end{array}\right)$. It is well known that $\sum_{i=0}^{n}\left(\begin{array}{c}n+1-i \\ i\end{array}\right)=F_{n+1}$ (and easily proven using induction and Pascal's Identity), where $F_{n}$ is the $n$th Fibonacci number. So $\mathcal{G}_{n}(1)=F_{n+1}$. From $\mathcal{G}_{n}(t)=\sum_{i=0}^{n}\left(\begin{array}{c}n+1-i \\ i\end{array}\right) t^{n-i}$ we get $\mathcal{G}_{n}{ }^{\prime}(t)=\sum_{i=0}^{n}(n-i)\left(\begin{array}{c}n+1-i \\ i\end{array}\right) t^{n-i-1}$, so $\mathcal{G}_{n}{ }^{\prime}(1)=\sum_{i=0}^{n}(n-$ i) $\left(\begin{array}{c}n+1-i \\ i\end{array}\right)$. Then:

$$
\mathcal{G}_{n+2}{ }^{\prime}(1)=\mathcal{G}_{n+1}{ }^{\prime}(1)+\mathcal{G}_{n}{ }^{\prime}(1)+F_{n+3} .
$$

From Lemma 1 we can use induction to show that:

$$
\mathcal{G}_{n}{ }^{\prime}(1)=\sum_{i=0}^{n}(n-i)\left(\begin{array}{c}
n+1-i \\
i
\end{array}\right)=\left(\sum_{i=1}^{n-1} F_{i} F_{n-i+1}\right)+F_{n} .
$$

(Lemma 2)

And from Lemma 2 and the formula $F_{n}=\frac{1}{\sqrt{5}}\left[\left(\frac{1+\sqrt{5}}{2}\right)^{n+1}-\left(\frac{1-\sqrt{5}}{2}\right)^{n+1}\right]$ we find that:

$$
\mathcal{G}_{n}^{\prime}(1)=\sum_{i=0}^{n}(n-i)\left(\begin{array}{c}
n+1-i \\
i
\end{array}\right)=\frac{n\left(F_{n+1}+F_{n+3}\right)-2 F_{n-1}}{5} \text {. }
$$

(Lemma 3)

For the sake of brevity, the proofs of the lemmas are not included here.

Finally we have that the average height of an $n$-step ussaw on the $[0,1]$ strip is:

$$
\frac{\mathcal{G}_{n}{ }^{\prime}(1)}{\mathcal{G}_{n}(1)}=\frac{n\left(F_{n+1}+F_{n+3}\right)-2 F_{n-1}}{5 F_{n+1}}
$$

\section{References}

[1] N. Madras and G. Slade, "The Self-Avoiding Walk," Birkhauser Boston, Woodbine, 1993.

[2] F. Roberts, "Applied Combinatorics," Prentice-Hall, Inc., Englewood Cliffs, New Jersey, 1984. 
[3] H. Wilf, "Generatingfunctionology," Academic Press, San Diego, 1994.

[4] D. Zeilberger, "Self-Avoiding Walks, The Language of Science, and Fibonacci Numbers." Available from Zeilberger's home page, http://www.math.temple.edu/^zeilberg/. 